\title{
Intermédialités
}

Histoire et théorie des arts, des lettres et des techniques

Intermediality

History and Theory of the Arts, Literature and Technologies

\section{(Dé)synchro-Shadok. Animation, musique concrète et ingénierie à l'ORTF}

\section{Sébastien Denis}

Numéro 19, printemps 2012

synchroniser

synchronizing

URI : https://id.erudit.org/iderudit/1012658ar

DOI : https://doi.org/10.7202/1012658ar

Aller au sommaire du numéro

Éditeur(s)

Revue intermédialités (Presses de l’Université de Montréal)

ISSN

1705-8546 (imprimé)

1920-3136 (numérique)

Découvrir la revue

Citer cet article

Denis, S. (2012). (Dé)synchro-Shadok. Animation, musique concrète et ingénierie à l'ORTF. Intermédialités / Intermediality, (19), 103-120.

https://doi.org/10.7202/1012658ar
Résumé de l'article

La série Les Shadoks de Jacques Rouxel, produite en France entre 1968 et 1974 , est emblématique de l'esprit expérimental de Pierre Schaeffer, à la tête à l'époque du Service de la Recherche de l'ORTF. Si la série est largement axée sur la désynchronisation entre image et son pour mettre en valeur la musique concrète, Schaeffer a pour sa part tenté de synchroniser ses équipes avec des découvertes technologiques (notamment l'animographe) tout en désynchronisant l'organisation de son service et en resynchronisant le public avec un esprit moderne, en rupture avec le conformisme audiovisuel des années Pompidou. 


\title{
(Dé)synchro-Shadok
}

\section{Animation, musique concrète et ingénierie à l'ORTF}

\author{
Sébastien Denis
}

\begin{abstract}
Plus ils pompaient dans l'autre sens, c'est-à-dire en reculant, eh bien plus non seulement ils ne reculaient pas, mais plus aussi ils n'avançaient pas non plus. Vous allez dire, dans ces conditions-là, ça aurait été aussi simple de ne pas pomper du tout. Ça prouve que vous n'y connaissez rien; car c'est un principe de base en pompologie: il vaut mieux pomper d'arrache-pied même s'il ne se passe rien plutôt que de risquer qu'il se passe quelque chose de pire en ne pompant pas.
\end{abstract}

Les Shadoks, $3^{\mathrm{e}}$ série, $4^{\mathrm{e}}$ épisode

ême quand on n'entend pas la voix de Claude Piéplu ${ }^{1}$, ces quelques fragments brassent la paralogique «shadok» dans une phraséologie qui, paradoxalement peut-être, a tout à voir avec les questions de (dé)synchronisation. Car, enfin, qu'est-ce que la pompologie ${ }^{2}$, si ce n'est la science de synchroniser tout un peuple avec le mouvement absurde du monde et de la science? C'est bien le paradoxe de cette série faite par des marins et des ingénieurs que de créer une mécanique huilée au service du nonsense... et de fabriquer de la communication intelligente à partir d'anti-communication, comme les shadoks créent leur planète à partir d'anti-matière. Ainsi la série parle-t-elle pour le contenant lui-même, le Service de la Recherche de l'ORTF (ci-après SR) ${ }^{3}$, à la manière d'une gigantesque métonymie des interactions image/son au service d'une nouvelle communication dans une ère technocentrée. Les trois séries des Shadoks produites entre

1. Claude Piéplu a été la seule voix over des Shadoks, adoptant un ton souvent professoral et décalé. Il est pour beaucoup dans le succès de la série.

2. A brief note for our english readers: the Shadok series is famous in France for at least one expression: «Et les Shadoks pompaient» (And the Shadoks kept on pumping), which has become a statement to evoke either the monotonous or the absurd moments in one's existence. "Pompologie» is thus the science of pumping.

3. Office de Radiodiffusion Télévision Française, créé en 1964 à la suite de la RTF et scindé en 1974 en plusieurs pôles. 

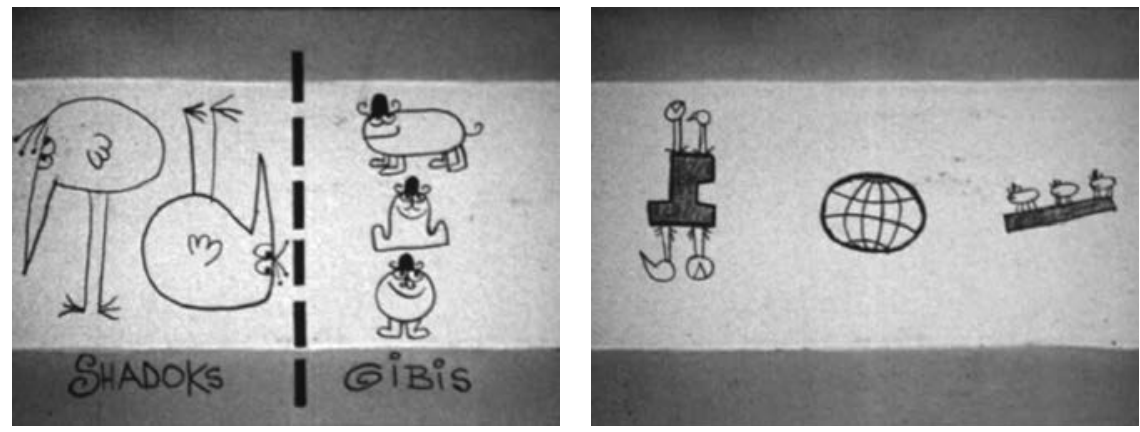

Fig. 1: Shadoks et Gibis. Les Shadoks, série 1, épisode 1, 1968 (gauche); Fig. 2: La planète Shadok, la Terre, la planète Gibi, ibid., épisode 2, 1968 (droite) (C) INA.

1041968 et $1974^{4}$ portent la marque du SR par leur volonté de casser l'image des programmes de flux habituels de l'ORTF. Elles le font en proposant un programme innovant, caustique, différent ${ }^{5}$. Il faut dire que le synopsis du réalisateur Jacques Rouxel avait, depuis 1965, de quoi laisser perplexes les directeurs du vénérable Office qui se succédèrent et en empêchèrent la diffusion jusqu'en 1967. Il y était question d'oiseaux bizarres (les shadoks) et de cochons d'Inde (les gibis) from outer space ${ }^{6}$ (Fig. 1) se débattant pour quitter leurs inhabitables planètes respectives (Fig. 2) afin de rejoindre la Terre (c'est l'argument de la première saison), où ils rencontrent son seul habitant, le terrible insecte Gégène ${ }^{7}$ (Fig. 3), avec lequel les shadoks se battent et les gibis s'amusent (deuxième saison), avant de retourner sur leurs planètes pour de nouvelles aventures (situées bien après les premières),

4. Une pré-série a été produite en 1967 (intégrée ensuite dans la première série intitulée Le Shadok), et une quatrième l'a été en 1999, diffusée sur Canal+. Chaque série comporte 52 épisodes de 2 ou 3 minutes.

5. Si beaucoup a été écrit sur Pierre Schaeffer, peu de chercheurs se sont penchés sur les Shadoks. Un ouvrage est en préparation aux Éditions du Chêne, mais il s'agit d'un «beau livre» sur l'œuvre de Jacques Rouxel, et non d'une analyse historique. Philippe Langlois leur dédie quelques pages dans Les cloches d'Atlantis. Musique électroacoustique et cinéma, archéologie et histoire d'un art sonore, Paris, Éditions MF, 2012; et on en trouve également une mention dans Évelyne Gayou, Le Groupe de Recherches Musicales. Cinquante ans d'histoire, Paris, Fayard, 2007. Mais il faut se pencher sur la revue Fantasmagorie, $\mathrm{n}^{\circ} 2$ 2, 1979, et sur L'abécédaire raisonné des Shadoks de Jean-Paul Dupuy (Paris, Nicolas Philippe, 2003) pour avoir plus de détails.

6. Gibi: «G.B.» prononcé à l'anglaise. Rouxel commence à travailler sur des «préshadoks » à partir de juin 1965, mais la commande officielle pour une série ne date que de juin 1966; INA, AREORI ooo12830INAo2.

7. Minuscule mais d'une puissance phénoménale, il peut représenter les forces de la nature. 

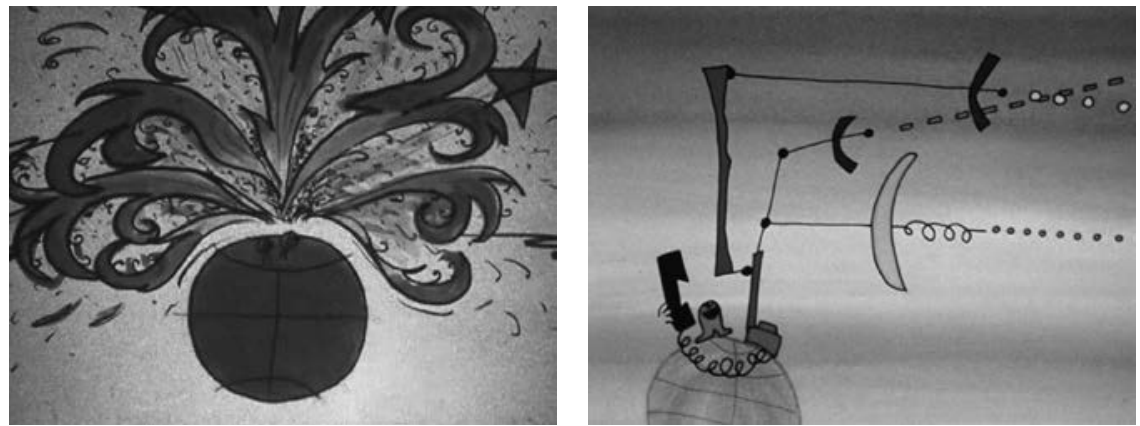

Fig. 3: Gégène en colère. Les Shadoks, série 1, épisode 52, 1968 (gauche); Fig. 4: Les moyens de communication gibis, ibid., série 3, épisode 1, 1974 (droite) (C) INA.

que vont connaître les arrière-arrière-arrière-petits-enfants des shadoks et gibis originels et qui seront retransmises grâce aux moyens de communication gibis (Fig. 4) (troisième saison). Les shadoks, vivant pour pomper (Fig. 5) et produisant des sons peu harmonieux, pondent des œufs en fer; les gibis, bien plus intelligents (Fig. 6), passent en fait leur temps à jouer des menuets très synchrones et communiquent grâce à leurs chapeaux.

Traditionnellement, la question du synchronisme est très importante en animation (même si elle est peu théorisée), car la réalisation image par image se fait le plus souvent en prenant pour point de départ la bande sonore - qu'il s'agisse de bruitages, de musiques ou de dialogues. S’agissant d'une série sans dialogues à l'image, accompagnée d'une musique, de bruitages et d'une voix over, Les Shadoks ont été entièrement sonorisés en postproduction. En amont, ils ont été largement inspirés à Rouxel par les styles visuels épurés du dessinateur Saul Steinberg et de l'animateur Stephen Bosustow (United Productions of America ${ }^{8}$ ). Les films UPA, qui veulent se démarquer de l'esthétique dominante Disney basée sur le réalisme, sont toutefois proches du cartoon traditionnel dans la réalisation des effets sonores et pratiquent une hyper-synchronisation image/ son/musique (un coup de poing/un bruit de gong, par exemple). Dès les années 1930, on appelle ce système le mickeymousing, pour moquer ce parti pris permanent d'analogie issu de Disney ${ }^{9}$ (et qu'on retrouve même chez un Norman

8. On doit à UPA notamment les séries Gerald McBoing Boing (sur un enfant ne s'exprimant que par le biais de bruits, klaxons, machines, etc.) et Mister Magoo. Leur esthétique est basée sur des traits nets, des décors suggestifs et des aplats de couleurs vives - à l'opposé donc du réalisme des productions Disney.

9. L'importance du musicien Carl Stalling, qui travaille dès 1928 avec Walt Disney, et ensuite avec l'équipe Warner, est extrême dans la définition d'une synchronisation image/ 

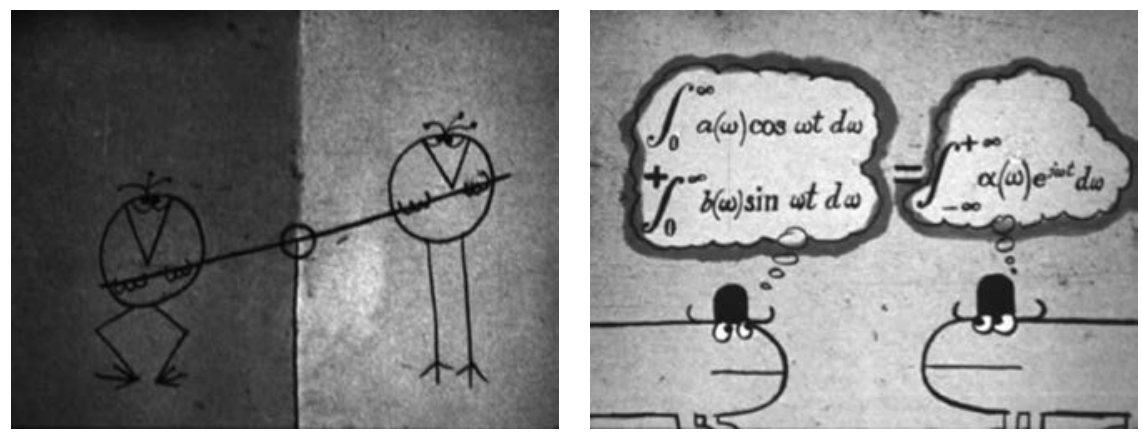

Fig. 5: Shadoks pompant. Les Shadoks, série 1, épisode 11, 1968 (gauche); Fig. 6: Gibis communiquant, ibid., épisode 3, 1968 (droite) (C) INA.

106 McLaren). Au contraire, la relation animation/musique au SR est basée sur une conception différente qui consiste à bannir la synchronisation systématique (que Michel Chion, issu du Groupe de Recherches Musicales [GRM], théorisera sous le terme de synchrèse ${ }^{10}$ ) de la plupart des compositions pour l'image, ce qui participe d'ailleurs d'une forme de flottement et d'anti-réalisme créant des «paysages sonores ». Alors que Les Shadoks est l'une des toutes premières émissions d'animation réalisées spécifiquement pour la télévision française de service public ${ }^{11}$, la série propose ainsi un modèle expérimental qui trouve son originalité dans un mélange de synchronisation cartoonesque et de désynchronisation entre image et son, joint à une volonté de diffuser la musique concrète - l'univers global créé par Rouxel ouvrant sur une possible lecture métaphorique du (dé)synchronisme.

\section{SYNCHRONISER ARTS, SCIENCES ET TECHNOLOGIES}

Pierre Schaeffer n'est pas l'initiateur principal des Shadoks: au sein du SR (créé en $1960^{12}$ ), c'est André Voisin, alors directeur des programmes, qui reçoit Jacques

son efficace, même si des expérimentations préexistaient largement à cette date (notamment les films sonores des frères Fleischer).

10. Il s'agit de la «soudure irrésistible et spontanée qui se produit entre un phénomène sonore et un phénomène visuel ponctuel lorsque ceux-ci tombent en même temps, cela indépendamment de toute logique rationnelle », Michel Chion, Le son, Paris, Nathan, 1998, p. 55 .

11. Ce sont surtout des séries américaines de cartoons qui sont alors diffusées à la télévision, la grande période de production de séries d’animation françaises ne commençant pas avant la fin des années 1970.

12. Pour une présentation générale de l'action de Schaeffer au SR, on se rapportera aux travaux de Jocelyne Tournet-Lammer : «Pierre Schaeffer et le Service de la Recherche de l'ORTF (1960-1974)», Hermès, nº 48, 2007, p. 79-86, et Sur les traces de Pierre Schaeffer, 
Rouxel en 1965 et qui instruit la cause de la série jusqu'à l'autorisation de diffusion donnée par Émile Biasini en 1967. Schaeffer n'a que très peu écrit sur les Shadoks, et pas davantage sur les nombreuses expériences d'animation dévelop-

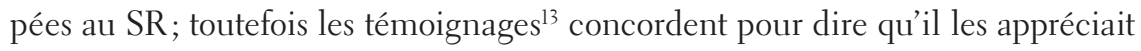
particulièrement et qu'il les a défendus auprès d'un ORTF peu enclin à favoriser ce type de programme. Il prône, de manière générale, une recherche audiovisuelle sur les plans narratif, esthétique, musical, mais aussi technologique, car c'est celui qui permet au projet d'advenir : c'est dans l'expérimentation permanente des sons et des images produits de manière concrète, grâce à des machines elles-mêmes concrètes, que se trouve non pas la solution, mais le questionnement autour d'une production audiovisuelle en constant renouvellement, en dehors des champs disciplinaires habituels. Comme Schaeffer le note pour la musique concrète, «il s'agira non d'une discipline, mais d'une interdiscipline, posant les problèmes dans une dimension originale, et n'ayant nul besoin des superpositions de solutions qu'on pourrait obtenir en confrontant les spécialistes de tel ou tel aspect partiel de la question ${ }^{14} »$.

L'interdiscipline appliquée aux Shadoks consiste en la rencontre a priori improbable entre un dessinateur issu de la publicité, ayant fait des études scientifiques mais nourri du Roman de Renart ( $12^{\mathrm{e}}$ et $13^{\mathrm{e}}$ siècles) ou de Jarry (Jacques Rouxel), un musicien classique passé à la musique concrète (Robert CohenSolal), un chef opérateur spécialisé dans l’ingénierie audiovisuelle (Pierre Mandrin, qui s'occupera ensuite de vidéo mobile), et enfin différentes personnes issues du champ plus traditionnel du dessin animé (notamment René Borg pour la première série); tout ce petit monde étant supervisé (d'assez loin) par un poète et conteur (André Voisin), un ingénieur en chef musicien (Pierre Schaeffer), ou pour les $2^{\mathrm{e}}$ et $3^{\mathrm{e}}$ séries, par une productrice «pop» issue de l'émission de variétés Dim Dam Dom (Manette Bertin). Cette interdiscipline émerge dans le cadre non directif d'une recherche basée sur l'emploi d'une technologie balbutiante (l'animographe, Fig. 7, et qui se fait dans des conditions proches du bricolage - ce sera moins vrai pour les $2^{\mathrm{e}}$ et $3^{\mathrm{e}}$ séries, réalisées par le Service de l'Animation de l'ORTF et donc financées en grande partie par l'Office, et pas seulement par le

Paris, INA/Documentation française, 2006. Merci à Jocelyne Tournet-Lammer pour son aide précieuse dans la recherche des témoins de cette période.

13. Des entretiens ont été réalisés pour cette recherche avec les témoins directs de l'aventure Shadoks dont les noms suivent: Manette Bertin, Régine Bezasse, Robert CohenSolal, Pierre Mandrin, Michel Marmin, Marcelle Ponti-Rouxel, Bernard Spehner.

14. Pierre Schaeffer, La musique concrète, Paris, PUF, coll. «Que sais-je?», 1967, p. 57 . 

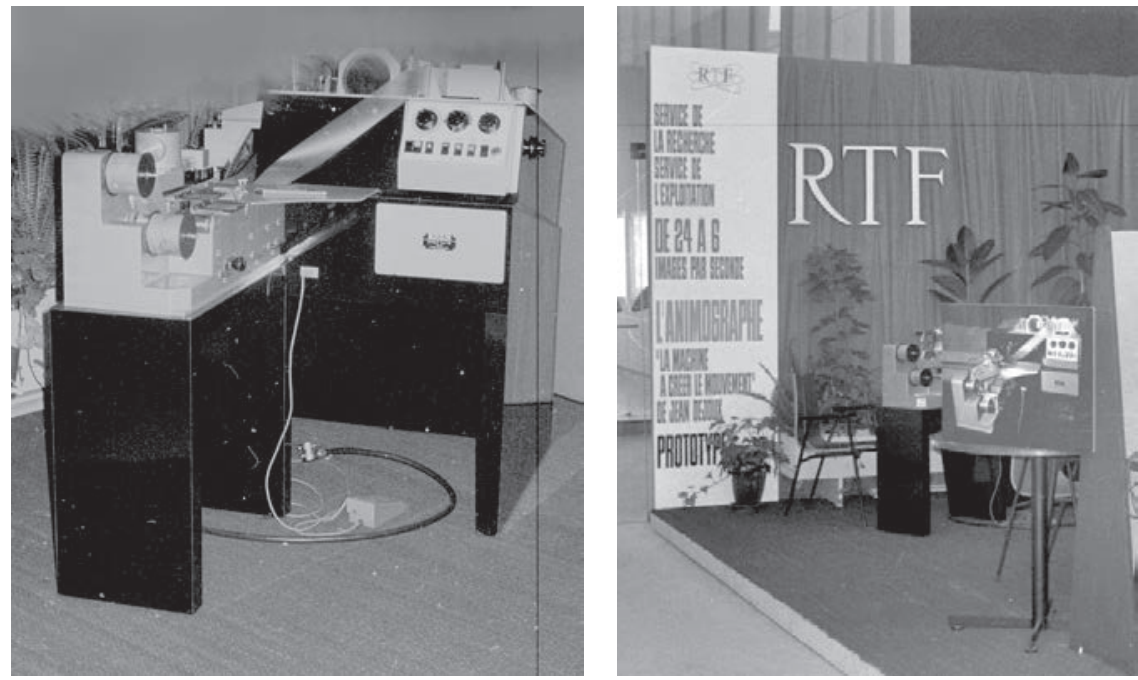

Fig. 7: Lanimographe. Photo Bernard Spehner, sans date (gauche); Fig. 8: Présentation de l'animographe au MIPE TV de Lyon 1963. Photo Bernard Spehner (droite).

SR. Ainsi, l'innovation technologique est garante de la recherche plus sûrement que l'innovation artistique, dont se méfie Schaeffer; d'où sa propension à mettre en doute son propre travail artistique et celui des autres au nom d'une recherche permanente qui ne saurait, en fait, jamais aboutir sous peine de n'être plus de la recherche ${ }^{15}$. Il créé un département spécial au sein du SR pour permettre à d'autres ingénieurs et scientifiques d'élaborer différents outils pour le traitement du son et de l'image: le Groupe de Recherches Techniques (GRT) fait écho au GRM et au Groupe de Recherches Image (GRI). Les techniciens du GRT développent leurs machines certes dans leur coin, mais avec l'obligation de travailler concrètement avec les chercheurs dans les domaines de l'image et du son. Les Shadoks ont été rendus possibles par une de ces machines infernales.

L'animographe, inventé par le dessinateur Jean Dejoux en 1961, mais construit par Francis Coupigny et son équipe du GRT en $1962-1963^{16}$, est un outil de rationalisation des méthodes et pratiques traditionnelles en animation. Dans l'esprit de Schaeffer, il faut profiter des améliorations de ce qu'une machine peut faire vite et bien, même si sa haine du «tout-machine» (il ne supporte pas

15. C'est aussi une des raisons pour lesquelles une fois la « recherche» sur Les Shadoks aboutie (1966-1968), la série doit trouver une place dans les programmes traditionnels de l'ORTF ou dans l'industrie.

16. Un contrat est signé en juillet 1962 pour cinq ans entre Dejoux et le SR; archives INA, AREORI 00012830 INAO2. 
la musique entièrement synthétique, non «concrète») empêche la création de machines qui n'auraient pas besoin de l'homme. À cet égard, l'animographe est passionnant: c'est une sorte de planche à dessin reliée à un appareillage qui, entre technologie pure et bricolage, permet de doubler le pouvoir créateur de l'artiste tout en conservant le côté manuel si important pour Schaeffer. Une fois un premier dessin appliqué sur une première bande de $70 \mathrm{~mm}$ grâce à la planche à dessin (et dupliqué par la machine), un autre est dessiné et dupliqué sur une seconde bande de $70 \mathrm{~mm}$, la machine se chargeant de faire une synthèse entre ces deux points clés, par le biais d'un filtre polaroïd, ce qui permet de ne dessiner en fait que 6 dessins à la place des 24 (ou 12) images traditionnelles en animation, comme l'ORTF l'annonce fièrement au MIPE TV de Lyon en 1963 (Fig. 8). C'est donc une limited animation ${ }^{17}$ qui est automatiquement transformée en full animation par interpolation automatique des phases manquantes. La réflexion et l'action entreprises par Dejoux, dessinateur et bricoleur plus qu'ingénieur, marquent la nette différence existant entre la France et la situation quasi hégémonique des studios américains:

Le dessin animé n'avait d'autre débouché que le spot commercial que l'on voit à l'entracte dans les cinémas à cause de son prix de revient extraordinaire, donc j'ai pensé - c'est élémentaire - que la télévision était le support idéal, par son format, par son moyen de diffusion, pour le dessin animé. Alors à ce moment-là, même raisonnement, je me suis plongé dans le problème Télévision, j’ai approché cette maison de la rue Cognacq-Jay [l'ORTF] subrepticement, par toutes les portes - et il y en a beaucoup - et j'ai commencé à réaliser que si on se penchait en face du problème en pensant Télévision/Animation, en oubliant que le système optique du cinéma existait, on pouvait trouver un raccourci, un joint ${ }^{18}$.

Dejoux indique que «Schaeffer et son état-major ont vu nos plans» et que le prototype Caroline a ensuite été réalisé afin d'« explorer toutes les possibilités » et d'établir «un plan de production qui touchera à tous les domaines de l'animation, du cinéma scolaire au cinéma de délassement, au cartoon, au cinéma

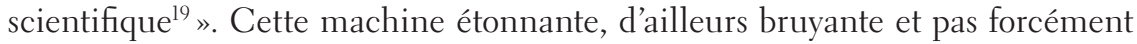
très fonctionnelle, a donc été créée spécifiquement pour la télévision en 1963

17. L'animation limitée (basée sur une réduction du nombre de dessins par seconde) est alors la technique dominante en animation, la montée en puissance de la télévision depuis les années 1950 ayant amené des coupes budgétaires importantes; voir Sébastien Denis, Le cinéma d'animation, Paris, Armand Colin, 2011, p. 148-149.

18. Au-delà de l'écran, ORTF, 10 février 1963, entretien de Jean Dejoux par Pierre Sainderichin.

19. Ibid. 
afin de dépasser le traditionnel banc-titre sur lequel se tournaient alors les dessins animés du monde entier. Une de ses premières applications, en 1965, est d'ailleurs une série de courts sujets réalisés en quelques heures pour le Journal Télévisé du soir. Pierre Mandrin, chef opérateur de formation, chargé de faire fonctionner l'animographe et de filmer les résultats ${ }^{20}$, indique que si personne (en dehors du SR) n'a cru en cette machine pourtant révolutionnaire (l'exemplaire de l'ORTF ne restant ainsi qu'un prototype), d'autres animographes parfaitement fonctionnels ont été construits de manière industrielle, en Italie ou en Amérique du Nord ${ }^{21}$. Or c'est bien parce que cet outil est resté à l'état de prototype qu'il a fini par rendre l'âme, Les Shadoks étant alors réalisés dès la $2^{\mathrm{e}}$ série (produite en 1969) en animation traditionnelle (celluloïd, encrage, gouachage) par le Service de l'Animation de l'ORTF.

La force initiale de la série tient dans l'adéquation entre d'une part l'outil et la simplicité des dessins proposés par Rouxel, et d'autre part le fond et la forme, ce qui fait des Shadoks une série technologique. Produite de manière artisanale sur un appareil inachevé, dans un moment de retour en force d'une dérision généralisée ${ }^{22}$, la série suit une paralogique qui conduit fatalement, dans le champ artistique mais aussi technologique, à une remise en cause de la logique elle-même et des fondements scientifiques solidement établis. D’ailleurs, des polytechniciens (le corps d'origine de Schaeffer) ne s'y trompent pas quand ils sont interrogés pour l'émission Les Français écrivent aux Shadoks en 1969, une émission qui rend compte des clivages générés par la série dans la société française: «On est tout à fait à l'aise quand il s'agit des Shadoks. Nous sommes devant notre poste de télévision, ça nous rappelle les mathématiques, ça ne nous sort pas de notre monde. [...] Les Shadoks nous rappellent la première démarche de la recherche. Nous sommes des mathématiciens, ce qui nous intéresse la plupart du temps, c'est la recherche imaginative d'un certain nombre de solutions et de concepts que nous imaginons. » Un autre enchaîne: «On en tire toutes les conclusions logiques, qui forcément arrivent à un résultat absurde qui est comique ${ }^{23}$. $\mathrm{La}$

20. Il fallait, en fait, retourner image par image chacune des images composites produites par l'animographe.

21. Entretien téléphonique le 7 avril 2012.

22. Voir à ce propos Bertrand Lemonnier, «L'entrée en dérision », Vingtième siècle, revue d'histoire, $n^{\circ}$ 98, 2008, p. 43-55. L'article ne fait que mentionner Les Shadoks, mais brosse un large portrait des formes dérisoires à cette époque dans la presse, le cinéma ou le spectacle vivant.

23. Les Français écrivent aux Shadoks, «L'avis des polytechniciens », ORTF, 25 février 1969. 
recherche schaefferienne est d'ordre techno-artistique, et non réellement scientifique. Il n'est donc pas très étonnant que la science-fiction soit si présente dans les travaux d'animation du SR, puisque ce genre ${ }^{24}$ permet justement de travailler directement la notion d'entropie.

\section{(DÉ-) SYNCHRONISER MUSIQUE ET IMAGE}

Pierre Schaeffer décrit ainsi la musique concrète en 1967: «Non seulement des sons jamais entendus, mais des assemblages de sons dont il était impossible de dire s'ils obéissaient à des lois prévues par les auteurs, ou s'ils tenaient simplement du hasard. Même envoûtant, ce nouveau langage était étrange, pour ne pas dire étranger. S'agissait-il même encore d'un langage ${ }^{25}$ ? » Et il s'interroge encore: «La révolution technique qui atteint la musique apporte-t-elle de nouveaux moyens de "faire de la musique" comme auparavant, ou bien nous mènet-elle à la découverte de nouvelles musiques, que nous ne savons pas encore faire, et encore moins entendre ${ }^{26}$ ? » Le but du SR est d'élargir cette conception à l'audiovisuel, et donc non pas de «faire des films » comme auparavant, pour paraphraser la proposition précédente. Il s'agit bien de rechercher de nouvelles formes audiovisuelles encore inconnues. Il est aisé d'envisager l'impact de la conception schaefferienne sur l'équipe des Shadoks qui travaille au même moment sur le prototype de la série. D'ailleurs, on note que plusieurs travaux du SR semblent précéder l'esthétique shadok, à commencer par le film Trois portraits d'un oiseau qui n'existe pas de Robert Lapoujade et François Bayle en 1963 (diffusé en janvier 1965), dans lequel les sons de mouettes modulés au début ressemblent étrangement aux couinements des shadoks. Le film est basé sur un poème de Claude Aveline, dont un extrait dit: «Le rêve d'un oiseau qui n'existe pas, c'est de ne plus être un rêve. Personne n'est jamais content. Et comment voulez-vous que le monde puisse aller bien dans ces conditions? » Dans ce film, comme dans beaucoup d'autres films d'animation produits au SR, on entend une composition

24. Guillaume Soulez indique à juste titre que la critique des genres par Schaeffer permet «de fabriquer des films qui ne préconstruisent pas les attentes spectatorielles d'une façon qui irait contre le travail de déconstruction appelé "déconditionnement" »; "L'effet-genre. Pratique et critique de la notion de genre au Service de la Recherche», dans Raphaëlle Moinde (dir.), Le cinéma français face aux genres, Paris, AFRHC, 2005, p. 72. On peut toutefois parler ici clairement d'un genre pour la SF, mais la place manque ici pour développer cette question.

25. Schaeffer, 1967, p. 5.

26. Schaeffer, 1967 , p. 7. 
sonore complexe, basée sur des séries de jeux d'(a-)synchronisme, comme on en retrouvera dans Les Shadoks.

Comme l'indique Pierre Schaeffer à Pierre Desgraupes en 1959 (dans son émission Lectures pour tous), «par rapport à la musique traditionnelle, la musique concrète, c'est un cinéma, c'est-à-dire un ensemble de sons qui sont assemblés comme les plans d'images. On photographie les sons sur bande magnétique, et ces sons présentent la souplesse que présentent les plans qui sont montés dans les films, avec des gros plans - on aperçoit tout à coup l'acteur, qu'on ne peut pas voir au théâtre, en gros plan, dont on aperçoit le détail du visage ou d'une expression, ou le rythme échevelé d'une séquence ${ }^{27}$. $\$ \mathrm{Si}$ on retrouve ce type d'éléments dans le Traité des objets musicaux (1966), dans lequel le son peut être envisagé comme une métonymie du cinéma ${ }^{28}$, on peut légitimement s'étonner du peu d'intérêt apparent que Schaeffer accorde à l'image pour elle-même alors qu'il est chargé de développer un service audiovisuel. Ce serait oublier un peu vite, comme le rappelle Philippe Langlois, que dans ses premiers travaux durant la Seconde Guerre mondiale, Schaeffer analyse la relation image/son comme étant centrale ${ }^{29}$. Mais c'est surtout par le biais de ses films pour la télévision qu'il va prendre à bras-le-corps ces interactions, et non par le biais de l'écrit - qu'on pense à Essais visuels sur les objets sonores (1962, diffusé en 1966), Essai sur la recherche image (1966) et Dialogue du son et de l'image (1967, diffusé en 1969), qui sont trois grammaires de l'image/son mettant particulièrement en valeur les expérimentations «concrètes» et l'animation reconstruisant un monde ex nihilo loin des «simulacres» que Schaeffer dénonce à la télévision ${ }^{30}$. Il faut donc, au moment de mettre en place le SR pour l'ORTF, valoriser «un certain cinéma qu'on pourrait appeler concret puisque de jeunes chercheurs comme Brissot, Laloux, Patris, s'efforcent de former des images comme nous avons cherché des sons $^{31} »$. Revenant, vingt ans plus tard, sur le Groupe de Recherches Image, il indique que

27. Lectures pour tous, RTF, 17 juin 1959.

28. Pierre Schaeffer, Traité des objets musicaux, Paris, Éditions du Seuil, coll. «Pierres vives ", 1966, p. 80-81.

29. Pierre Schaeffer, Essai sur la radio et sur le cinéma, Paris, Allia, 2010.

30. Pour Guillaume Soulez, c'est l'ensemble de la production du SR qui participe de ce mouvement dans un cadre pré-soixante-huitard; Guillaume Soulez, «Mai 68 avant Mai 68 au Service de la Recherche», Médiamorphoses, numéro hors-série «Les Empreintes de Mai 68 », n² 2008, p. 63.

31. Discorama, RTF, 12 juin 1959, Schaeffer interviewé par Jean Desailly. 

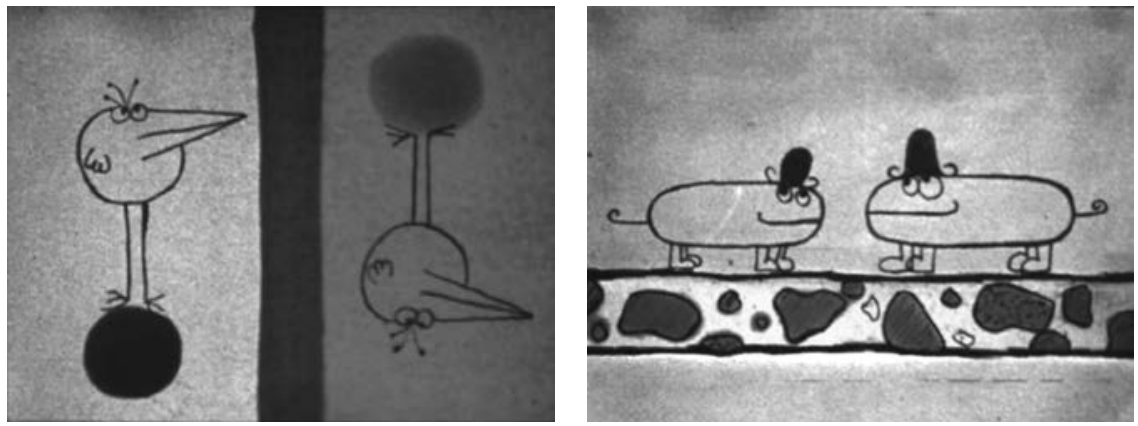

Fig. 9: Shadoks du haut et du bas. Les Shadoks, série 1, épisode 1, 1968 (gauche); Fig. 10 Gibis, ibid., épisode 1, 1968 (droite) (C) INA.

des gars (...) prétendaient faire avec des « objets visuels» ce que nous faisions avec les objets sonores: au lieu d'ébranler les tôles, de frotter les membranes, de réaliser des staccatos avec du scotch, ils allaient exploiter les phénomènes lumineux, les condensations animées de rayons réfléchis sur du clinquant (ce qu'on nomme des «caustiques» en classe de seconde), l'agitation des poussières sur un trembleur électrique ou encore les tracés de la limaille aimantée. Je ne pouvais renier ces disciples inattendus, tout en m'interrogeant sur leur insertion improbable dans les programmes de télévision. Gérard Patris et Jacques Brissot devinrent ainsi les fondateurs du groupe, bientôt rejoints par des virtuoses de l’ «image par image» et de la pellicule grattée ${ }^{32}$.

Les shadoks sont «concrets » car ils sont composés de formes géométriques: trois cercles, un triangle, deux lignes pour les jambes et six pour les pattes (Fig. 9). Les gibis sont des rectangles émoussés sur pattes surmontés de chapeaux (Fig. 10). La série se présente donc comme l'articulation de syntagmes visuels basiques assemblés pour créer une narration, cette simplification ultime des formes étant due à la taille de la table à dessin de l'animographe $\left(5 \mathrm{X}_{7} \mathrm{~cm}\right)$. Si les shadoks et les gibis sont dessinés par l'homme, ils renvoient à l'idée d'animaux de synthèse. À un retour à un solfège prenant pour base la réalité de sons de toutes sortes réaménagés en une nouvelle manière d'écouter les bruits du monde, Les Shadoks proposent un équivalent visuel : la simplification des formes correspond à une nouvelle manière de regarder le monde. La langue shadok elle-même fait figure de solfège préhistorique composé de quatre sons concrets GA BU ZO MEU (Fig. 11), déclinés ensuite à l'infini en fonction des situations (comme les cris du Marsupilami, créé en 1952). On pense à cette «musique de Néandertal» que Schaeffer analyse dans son Traité des objets musicaux: «L'homme préhistorique ne connaît-il pas ainsi un double usage de la voix : émettre des cris d'appel,

32. Pierre Schaeffer, Les antennes de Jéricho, Paris, Stock, 1978, p. 179. 
de détresse, de menace ou de colère, ou bien mettre à l'essai ce que les spécialistes appellent pompeusement son appareil phonatoire, plaisir de crier à pleins poumons, plaisir aussi de frapper sur des objets, sans que soient nécessairement dissociés le geste et son effet, la satisfaction d'exercer ses muscles et celle de "faire du bruit"33 ? »C'est bien cette double logique que met en œuvre la série à travers la notion de contrepoint, une notion développée par Schaeffer dans ses trois films déjà mentionnés. Il ne faudrait pas en effet faire penser au lecteur que seule une logique de désynchronisme serait à l'œuvre. Comme on l'a dit plus tôt, le modèle de référence est bien le cartoon UPA, jouant d'un synchronisme « décalé », c'està-dire d'effets synchrones employant des sonorités «concrètes », donc rarement usitées. Même si on ne les comprend pas, les shadoks «parlent» leur langue de manière synchrone et génèrent des sons synchrones en se frappant, mais tous ces sons ont été retravaillés de manière « concrète » de façon à créer un contrepoint.

C'est justement une des avancées de Robert Cohen-Solal, le musicien des Shadoks, que d'avoir tenté une forme plus populaire de contrepoint. L'usage de sons synthétiques est un des ingrédients capitaux des Shadoks, notamment pour le traitement sonore des gibis et de Gégène. Les shadoks de leur côté sont, de manière emblématique, sonorisés grâce à des voix humaines (en particulier la voix du frère du musicien, Jean Cohen-Solal, qui possède une sorte de troisième corde vocale) qui sont ensuite retraitées. Robert Cohen-Solal est au GRM depuis deux ans quand on lui propose le projet Shadoks en 1967. Remplaçant un premier musicien, Philippe Beetz, Cohen-Solal a déjà une expérience en musique concrète: après un stage de six mois en 1965, il est devenu l'assistant de Bernard Parmegiani, pour lequel il effectue un travail de préparation, de classement et de montage des sons dont Pierre Schaeffer indique la très grande importance dans la pensée «concrète » que reconnaît d'ailleurs Cohen-Solal:

Avant toute manipulation, on devra, pour commencer, mettre de l'ordre dans la moisson des objets sonores: présenter clairement les objets sonores intéressants, rejeter les autres, regrouper les familles. On apprendra ainsi, pour ces montages élémentaires, à se servir des ciseaux, du scotch et des amorces de différentes couleurs. Mais on n'en restera pas à ces simples travaux ménagers : le montage est sans doute, parmi toutes les opérations de la musique concrète, celle qui en illustre le mieux l'attitude nouvelle consistant à travailler au sein même de la matière sonore : le découpage possible à n'importe quel moment du son, le collage des fragments les plus disparates, le travail sur des sons «à l'envers » (que l'on fait commencer par la fin) manifestent en effet l'exercice d'un pouvoir sur le déroulement temporel des objets ${ }^{34}$.

33. Schaeffer, 1966, p. 42 (je souligne).

34. Schaeffer, 1967, p. $45-46$. 
C'est grâce à cette expérience fondamentale que Cohen-Solal réalise ensuite ses premiers travaux en tant que musicien pour plusieurs films d'animation, notamment Le trou (1968) de Piotr Kamler. Mais il rencontre au même moment un problème avec Les Shadoks, comme il l'indique dans un entretien ${ }^{35}$ : il lui faut respecter l'esthétique du GRM et du SR, tout en se concertant avec Jacques Rouxel, qui est pour sa part globalement rétif à la musique électro-acoustique et davantage porté sur le cartoon américain de style UPA déjà évoqué. On peut dire que si dans Les Shadoks la science-fiction passe par la musique électroacoustique a-synchrone, l'absurde vient de la répétition et de la synchronisation. Sur ce plan, les Shadoks sont donc très marginaux dans la production du SR, notamment du fait de Jacques Rouxel lui-même, et aussi parce que c'est la seule série animée de type cartoon au sein du SR... En plus, pour les gibis en particulier, Cohen-Solal emploie une forme musicale clairement éloignée de la musique concrète, basée sur des leitmotive que sa formation classique au Conservatoire de Nîmes mais aussi son amour des Beatles lui permettent d'aborder sans problème (on pense aux menuets dans la $2^{\mathrm{e}}$ série en particulier), et il le fait avec des instruments dans l'air du temps (flûte traversière, groupe pop: basse, batterie, guitare). Pierre Schaeffer non seulement ne s'y oppose pas, mais applaudit des deux mains $^{36}$, à la surprise du musicien. Cohen-Solal aborde cependant les trois séries d'une manière différente : il travaille sur la première série en 1967-1968 avec peu de moyens et dans des conditions précaires, sur la seconde en 1969-1970 avec plus de moyens et d'instrumentistes, et sur la troisième en 1974 avec à nouveau très peu de moyens, et cette fois-ci chez lui dans une ferme isolée, à partir d'instruments électroniques (il a en effet quitté le SR en 1973).

\section{(DÉ)SYNCHRONISER LE SERVICE DE LA RECHERCHE}

C'est son propre service que dès sa création Schaeffer décide à la fois de synchroniser et de désynchroniser, avec un mode opératoire qui a pu sembler brutal à

35. Entretien téléphonique le 4 avril 2012.

36. Il parle des «impayables Shadocks [sic] de Rouxel» dans Schaeffer, 1978, p. 194; et il donne à la série «un rôle de réanimation à petites doses qui, à la longue, entraînera un renouvellement de ton et de style dans les autres émissions», cité dans Sylvie Dallet (dir.), Pierre Schaeffer. Itinéraires d'un chercheur, Montreuil, CERPS, s.d., p. 67. 
certains $^{37}$. En bon boy scout (ce qu'il a été dans sa jeunesse ${ }^{38}$, et le mot français éclaireur ne rend pas totalement compte de la dimension pionnière et exploratrice du boy scout), Schaeffer tente en effet d'une part de déstabiliser l'institution pour la faire évoluer, et d'autre part de créer un principe organisationnel basé sur le transfert de responsabilité, tout cela dans un service composé de « chercheurs » qui restent des artistes et n'ont aucun goût ni pour l'organisation ni pour les conflits inhérents au management hard mis en place par le directeur. L'organigramme du SR change perpétuellement pour empêcher les habitudes de s'installer, au risque d'une désorganisation jugée elle-même créatrice par Schaeffer. À voir les diagrammes employés par lui dans ses conférences publiques, on comprend qu'un ordre destructeur, qu'un principe d'entropie créatrice est à l'œuvre chez Schaeffer (à la fois brouillon et méthodique jusqu'à l'intransigeance). Ce principe doit nécessairement se retrouver dans son service, chez ses collaborateurs et dans leurs œuvres. Sur le plan métaphorique, l'univers des Shadoks pourrait-il être envisagé comme une représentation de l'univers du SR? Ce n'est qu'une intuition, mais elle est confirmée par Bernard Spehner ${ }^{39}$, ainsi que par Évelyne Gayou ${ }^{40}$. La planète Gibi, que ces petits personnages quittent d'ailleurs pour la Terre, car c'est une sorte de planche basculant d'un côté ou de l'autre dans le vide, fait penser au SR où l'insécurité était constante, à lire ou à écouter les témoignages. Les différentes planètes construites successivement par les shadoks sont, elles aussi, assez proches du capharnaüm que décrit André Voisin en 1966 quand il évoque le SR, alors même que Rouxel fait ses premières expériences sur l'animographe:

C'était ce formidable bordel de tables, de chaises, de lutherie avortée, d'écrans crevés, de foutoir de la rue de l'Université! Des salles de bains transformées en bureaux,

37. Voir notamment le film Les chercheurs 62, RTF, 1962, qui dresse un portrait peu avenant du SR tout en prouvant la grande capacité d'autocritique de Schaeffer.

38. «Je l'ai avoué et je persiste: j’en fus. Contraint comme on l'a vu de recourir aux basses classes d'âge, abandonné des cadres et l'esprit toujours un peu joueur, j’imaginais donc, poursuivant en cela mon périple adolescent, d'instaurer le système des patrouilles dans ma place assiégée. On ne peut savoir à quel point cette inoculation, apparemment inoffensive, se révéla contre-nature et dangereusement subversive», Pierre Schaeffer, Machines à communiquer. Tome 2. Pouvoir et communication, Paris, Éditions du Seuil, coll. «Pierres vives», 1972, p. 101-102.

39. Entretien téléphonique le 3 avril 2012. Bernard Spehner est responsable technique de l'installation des studios vidéo et de l'animographe durant la première série.

40. «Le scénario peut être compris comme une métaphore du "non-sens(e)" de la vie moderne dans nos sociétés industrielles et peut-être aussi à l'intérieur même du Service de la Recherche », Gayou, 2007, p. 145. 
des cuisines en corridors de confidences, l'hôtel particulier tout entier envahi par l'audiovisuel, en bazar, en gésine! (...) Le facteur humain prend une place considérable dans cette maison, et les gens sont finalement moins urbains entre eux que dans bien des endroits où l'on peut travailler: ils sont traqués. Étant constamment en état d'instabilité, ils projettent sur autrui une grande part de leur instabilité ou de leur agressivité ${ }^{41}$.

C'est une recherche de type fondamental qui est visée, mais elle doit impliquer une dimension psychologique afin que le SR n'entretienne pas des artistes, mais soutienne au contraire des «chercheurs » (typologie jamais employée auparavant pour des artistes), le but étant d'être crédible auprès de l'administration de l'ORTF². Pour Schaeffer, «l'important est tout d'abord de surprendre le chercheur, non seulement dans l'incertitude, mais dans l'ambiguïté de sa démarche ${ }^{43}$ ». Dans le célèbre livre Machines à communiquer, on trouve le témoignage de 1962 de Paule Sengissen sur son arrivée au SR: "J'entrais dans un truc qui tenait de Kafka et du stalinisme. J'allais faire quoi là-dedans? Je voulais bien faire quelque chose, mais quoi ${ }^{44}$ ? » Et celui de 1960 de Sophie Brunet, qui fut l'assistante personnelle de Schaeffer : «Le Service de la Recherche existe à la façon d'une œuvre d'art. [...] Il a, en commun avec cette œuvre, d'être là, indépendamment de ses buts, d'être unique, d'être irracontable, intraduisible dans un autre langage. Cette analogie éclaire certaines choses: les personnages qui le composent ne le comprennent pas plus qu'une touche de jaune ou de rouge ne comprend le tableau dans lequel elle se trouve ${ }^{45}$. " Plus tard, dans le chapitre qu'elle écrit, cette fois en 1972, dans le livre de Schaeffer, Brunet poursuit: «Les directives sont périmées au moment même où elles sont formulées, par le seul fait qu'elles ont été formulées. Toujours essoufflés, toujours en retard, nous courons après une situation qui nous dépasse ${ }^{46}$. » Il ne s'agit pas de dire que Les Shadoks seraient une image directe que Jacques Rouxel donnerait du SR, mais il n'est pas impossible que l'ambiance générale du Service ait quelque peu déteint sur l'ensemble de l'équipe, au point que les situations ubuesques dans

41. Entretien avec Lia Lacombe, dans Schaeffer, 1972, p. 118-120.

42. C'est vrai jusqu'aux contrats des «chercheurs », très particuliers mais que Schaeffer a réussi à obtenir de l'ORTF; entretien avec Régine Bezasse, administratrice du SR, le 2 avril 2012 à Aix-en-Provence.

43. Entretien avec Lia Lacombe, dans Schaeffer, 1972, p. 119.

44. Ibid.

45. Schaeffer, 1972, p. 121.

46. Ibid., p. 123. 
lesquelles Schaeffer/Shadoko ${ }^{47}$ plongeait ses chercheurs ont pu transparaître dans le mélange de désorganisation shadok et d'organisation gibi.

Ce qui se dégage à la fois des archives, des (nombreux) écrits de Schaeffer et des entretiens avec les témoins de cette période ${ }^{48}$, c'est paradoxalement l'absence des films eux-mêmes dans la théorie issue du SR. Les œuvres semblent émerger de la structure, presque sans concertation, du fait même qu'elles sont faites collégialement dans le respect d'une recherche basée sur une pratique concrète. Philippe Langlois insiste à juste titre sur le fait que dans les deux volumes des Machines à communiquer, «il n'est jamais fait mention d'une œuvre précise, d'un réalisateur, d'un compositeur, ou d'une réussite audiovisuelle (...) comme si les œuvres n'étaient au fond que les instruments d'une théorie plus globale visant à appréhender la question de l'expression à travers l'outil technologique ${ }^{49}{ }$. Mais l'absence de traces ou de souvenirs quant à la conception des œuvres semble surtout rendre compte de la très grande liberté dont jouissent les artistes dans le Service; en effet, les $\operatorname{archives}^{50}$ ne conservent que la mémoire de ce qui n’a pas fonctionné (défaillances techniques et organisationnelles), mais jamais d'une quelconque injonction artistique: c'est en effet le principe même de Schaeffer que de laisser libres ses chercheurs - lui étant libre de ne pas diffuser les résultats:

En affirmant une démarche expérimentale en matière d'art et une méthode interdisciplinaire, je faisais passer au second plan l'esthétique et sa proverbiale gratuité. En une époque moins baroque, moins hagarde, moins gâcheuse d'outils et d'auteurs, j'aurais pu avoir des scrupules. Mais en fait, lorsqu'un véritable talent se manifestait, il répondait spontanément à nos partis pris, il honorait sans difficulté le triple contrat

47. Une comparaison des schémas dessinés par l'un et par l'autre est parlante; mais les formules mathématiques des gibis sont aussi à prendre en compte. Le professeur Shadoko est le savant des shadoks. Il se livre à toutes sortes d'expériences mécaniques, chimiques ou mathématiques, le plus souvent vouées à l'échec. Une comparaison des schémas dessinés par Shadoko et par Schaeffer est parlante; mais les formules mathématiques (beaucoup plus efficaces) des gibis sont également à prendre en compte.

48. C'est, du fait du faible volume d'archives conservées (notamment à l'IMEC où l'énorme fonds Schaeffer ne contient étrangement aucune trace des Shadoks - merci pour leurs vérifications à Jacqueline Schaeffer et Martin Kaltenecker), beaucoup sur des témoignages que se base notre recherche actuelle.

49. Philippe Langlois, «Pierre Schaeffer et l'aventure de l'image», dans Martin Kaltenecker et Karine Le Bail (dir.), Pierre Schaeffer, les constructions impatientes, Paris, CNRS Éditions, 2012.

50. Les archives consultées pour cette recherche sont celles de l'INA, AREORIooo1283oINAoor à 003 (fonds Shadoks) et AREORIoo13515INAo1o (Service de la Recherche, carton 10: dossier animographe). 

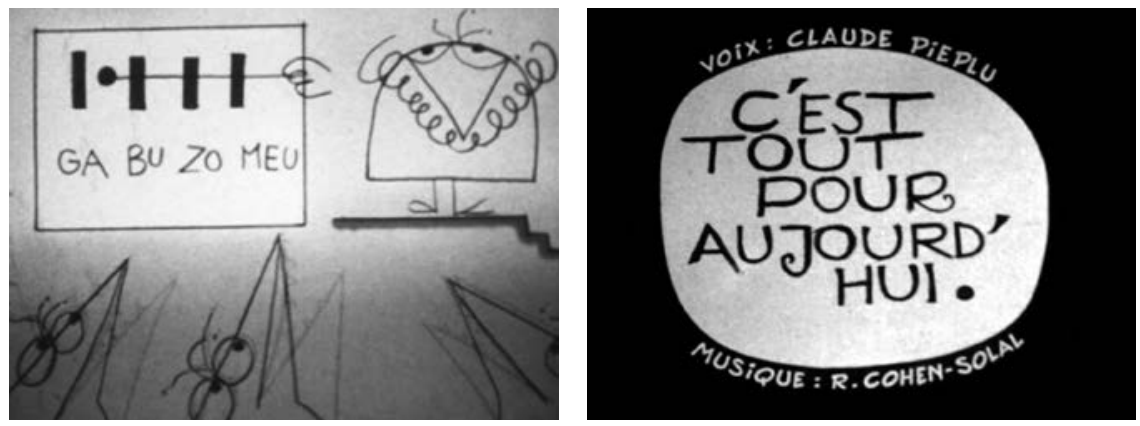

Fig. 11: Le professeur Shadoko en pleine leçon. Les Shadoks, série 1, épisode 49, 1968 (gauche); Fig 12: C'est tout pour aujourd'hui. Carton final des trois séries (droite) (C) INA.

de la technologie, de l'expression et de l'investigation psychologique, voire phénoménologique. Il me faut citer ici Piotr Kamler, Peter Foldès, Jacques Rouxel (ce dernier plus populaire en raison des Shadoks). Il pourront dire, si on les questionne, avec quel scrupule a été respectée leur responsabilité ou leur vocation d'auteurs. En fait, nous n'étions pas situés n'importe où, dans quelque Museum of Modern Art ou quelque officine d'avant-garde. Même si nous diffusions à la télévision aux heures de moindre écoute, nous n'en tenions pas moins à honorer, jusque dans ce même secteur, le cahier des charges des mass media. À l'inverse de ce qu'attendaient de moi tant d'artistes ou se disant tels, nous n'étions nullement obligés d'assurer, coûte que coûte, la diffusion d'essais douteux, insoucieux de tout public ${ }^{51}$.

Il s'agissait donc in fine, tout en leur laissant une entière liberté de travail, de synchroniser les «chercheurs » avec le fonctionnement d'une administration et avec le cahier des charges de l'audiovisuel public au service des téléspectateurs. Quant à la relation de Schaeffer avec ces derniers, elle visait à les désynchroniser de leurs habitudes ronronnantes dans un geste qui était somme toute assez politique en 1967. Si Les Shadoks ont eu un tel impact sur la France de la fin des années 1960 (de nombreux commentateurs ont noté que la série a été diffusée à partir de la fin avril 1968, soit quelques jours avant le début des manifestations estudiantines du Quartier latin ${ }^{52}$ ), c'est probablement parce qu'ils tendaient aux spectateurs de la France de Pompidou et des Trente glorieuses un miroir incer-

\section{Schaeffer, 1972, p. 155-156.}

52. Au point que la série est devenue culte pour son aspect contestataire, alors que Rouxel lui-même refuse pour sa part cette étiquette. Il semble en effet avoir tenu une ligne anarchiste sans étiquette durant toute sa vie, comme le confirme sa femme (qui était, elle, très engagée à gauche). On verra sur ce point le documentaire de Jérôme Lefdup, Les Shadoks, mythe ou légende? (1999), dans lequel on note l'importance qu'ont eu Les Shadoks sur l'esprit de Canal+ dans les années 1980 et 1990. 
tain, polysémique mais brutal de la société de consommation et de communication. Comme le note rageusement Schaeffer,

La télévision est finalement la maquette d'un pays, le modèle réduit de notre société. Le spectacle qu'elle offre, sur tous les écrans du monde, coïncide à peu de chose près avec la pièce qui se joue ici et là. (...) Dans tous les pays du monde, la télévision est fidèle: elle est par construction le reflet obligé de ce qui s'y passe (elle manque aussi de ce qui manque). Le comble, c'est de la vouloir objective (objective avec quoi et pour qui?). Objective, elle l'est assurément, ajustée aux contraintes mutuelles de tous ceux qui sont là, tous ensemble, à vivre comme ça, à ne rien vouloir de plus, à se contenter de ça. Dis-moi quelle est ta télévision, je te dirai qui tu es. Il n'y a pas de mauvaises télévisions, il n’y a que des pays médiocres, en mal de civilisation, qui détestent leur miroir ${ }^{53}$.

Mais cela, comme on dit dans les contes, est une autre histoire - et donc, pour reprendre la chute quotidienne des Shadoks, «C'est tout pour aujourd'hui » (Fig. 12). 